Case Report Journal of Epilepsy Research pISSN 2233-6249 / elSSN 2233-6257

Received June 27, 2017

Accepted November 8, 2017

Corresponding author: Young-Soo Kim Department of Neurology, Gyeongsang National University Hospital, Gyeongsang National University School of Medicine, 79 Gangnam-ro, Jinju 52727, Korea

Tel. +82-55-750-9876

Fax. $+82-55-755-1709$

E-mail; youngsookim0127@gmail.com

\title{
Successful Use of Therapeutic Hypothermia for Refractory Nonconvulsive Status Epilepticus
}

\author{
Do-Hyung Kim ${ }^{1}$, Hye-hoon Kang ${ }^{1}$, Minjung Kim', Tae-Won Yang ${ }^{2}$, Oh-Young Kwon', \\ Jung Sook Yeom ${ }^{3}$, Bong Su Kang ${ }^{4}$, Yong Hwan Kim ${ }^{5}$ Young-Soo Kim ${ }^{1}$ \\ ${ }^{1}$ Department of Neurology, Gyeongsang National University Hospital, Gyeongsang National University School of \\ Medicine, Jinju; ${ }^{2}$ Department of Neurology, Changwon Gyeongsang National University Hospital, Gyeongsang \\ National University School of Medicine, Changwon; ${ }^{3}$ Department of Pediatrics, Gyeongsang National University \\ Hospital, Gyeongsang National University School of Medicine, Jinju; ${ }^{4}$ Department of Neurology, Yangpyeong \\ Hospital, Yangpyeong; ${ }^{5}$ Department of Emergency Medicine, Samsung Changwon Hospital, Sungkyunkwan \\ University School of Medicine, Changwon, Korea
}

\begin{abstract}
Therapeutic hypothermia $(\mathrm{TH})$ has rarely been utilized as an adjunct to anticonvulsants in treating patients with refractory convulsive status epilepticus (CSE). However, determining the effectiveness of $\mathrm{TH}$ in CSE is difficult due to the unavoidable use of sedative drugs to manage hypothermia. Additionally, the effectiveness of $\mathrm{TH}$ has not been studied in patients with refractory non-convulsive status epilepticus (NCSE). Here, we report the successful use of TH without additional sedative drugs in a patient with temporal lobe epilepsy and refractory NCSE. A 46-year-old man was referred to the neurology department because of recurrent seizure attacks. Electroencephalography (EEG) after first-line status treatment showed continuous periodic discharges consistent with NCSE. He was started simultaneously on continuous EEG monitoring and $\mathrm{TH}$, but was not administered any benzodiazepines to control shivering or maintain TH. During TH, EEG abnormalities gradually improved, and the patient regained consciousness in accordance with the improvement in EEG. The patient was alert and his EEG had normalized a few days after starting $\mathrm{TH}$. To the best of our knowledge, this is the first report describing the successful treatment of refractory NCSE with TH. As no sedative drugs were used during the maintenance of hypothermia, NCSE control may have been achieved by TH alone. (2017;7:109-114)
\end{abstract}

Key words: Hypothermia, induced, Status epilepticus, Electroencephalography

\section{Introduction}

Nonconvulsive status epilepticus (NCSE) is defined as a change in mental state from baseline, lasting at least 30-60 minutes, associated with continuous or near-continuous epileptiform discharge on the electroencephalogram (EEG). ${ }^{1}$ NCSE is thought to constitute 20-25\% of all episodes of status epilepticus (SE). ${ }^{2}$ Refractory SE (RSE) has been associated with high morbidity and mortality rates, and does not always respond to standard pharmacological treatments. When these treatments fail, more active and aggressive alternative treatments are required.

Systemic therapeutic hypothermia (TH) has shown benefits in various clinical settings, including post-cardiac arrest and neonatal hypoxic encephalopathy, and has been found to control intracranial pressure in patients with traumatic brain injury and stroke.-5 Experimentally, TH was shown to have anticonvulsant and neuroprotective effects after $\mathrm{SE}^{6-7}$ Although several case reports have shown promising results following the application of $\mathrm{TH}$ in patients with RSE, ${ }_{1}^{8-10} \mathrm{TH}$ was used as adjuvant therapy in almost all these patients. This report describes the successful use of TH without additional sedative drugs in a patient with temporal lobe epilepsy who experienced refractory NCSE. Findings in this patient suggest that TH can be considered an adjunctive or alternative therapy in patients with medically intractable NCSE.

\section{Case}

A 46-year-old man was referred to the neurology department of 


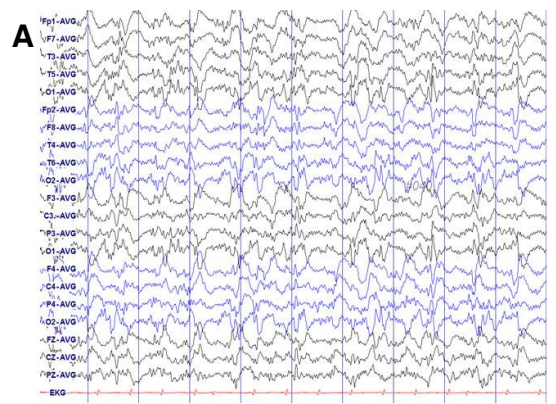

B
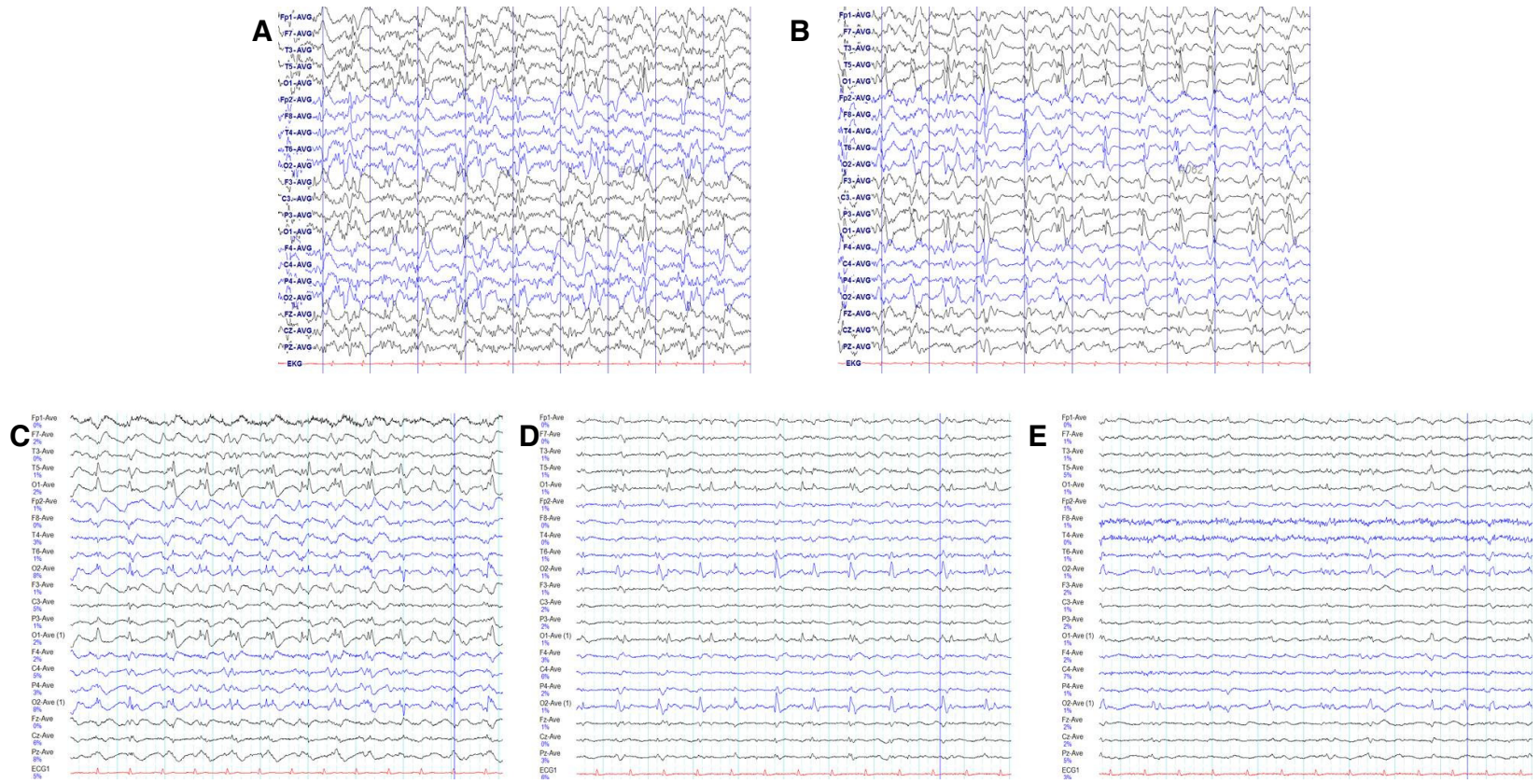

Figure 1. Ten-second EEG trace recordings with a 10-20 electrode system (A) following initial treatment of convulsive status epilepticus with lorazepam and fosphenytoin loading and showing continuous quasi-periodic single or poly-spikes and waves of high amplitude, mainly in both occipitoparietal areas; (B) after phenobarbital loading and just before induction of $\mathrm{TH}$; (C) following induction of hypothermia to the $35^{\circ} \mathrm{C}$ target temperature; and after (D) 12 hours and (E) 24 hours of hypothermia at $35^{\circ} \mathrm{C}$. EEG, electroencephalography.
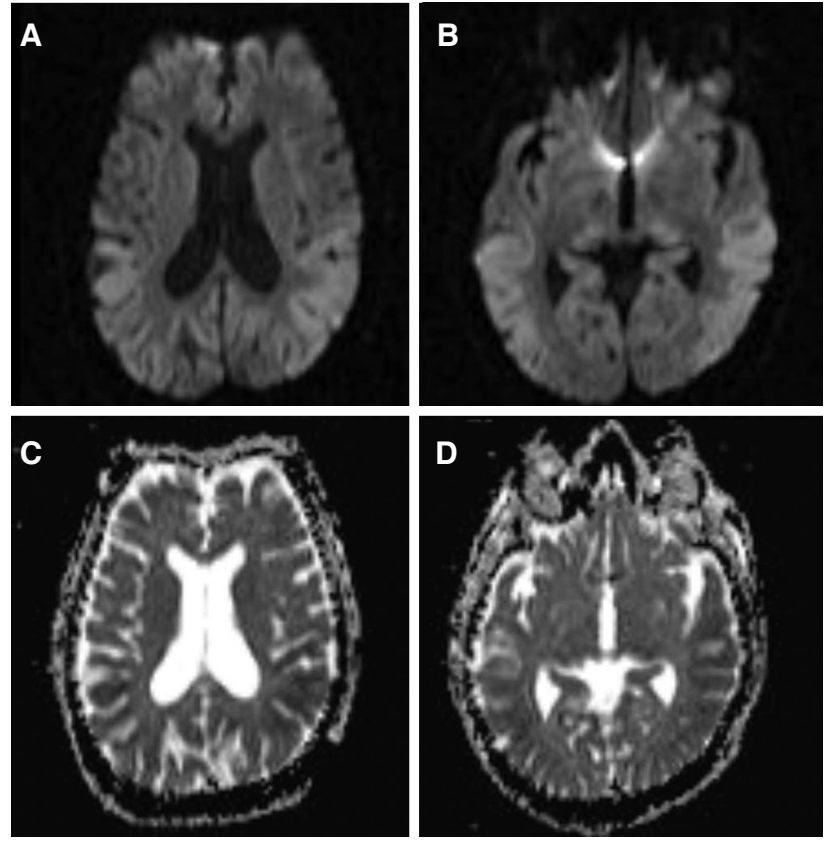

Figure 2. Brain (A, B) diffusion MRI and (C, D) ADC maps after initial treatment for convulsive status epilepticus. Both showed symmetric restricted diffusion in bilateral occipitoparietal areas. MRl, magnetic resonance imaging; ADC, apparent diffusion coefficient.

Samsung Changwon Hospital because of recurrent generalized tonic-clonic (GTC) seizures. At that time, the patient was undergoing wound care at the Orthopedics Department of our hospital because of necrotizing fasciitis of the right foot. GTC seizures were observed twice by station nurses. The duration of each seizure was slightly over 5 minutes, with the patient not recovering mental status between seizures. After the second GTC seizure, the patient was administered a loading dose of lorazepam, but he remained unconscious, and remnant subtle motor components were observed. The patient's caregiver informed us that the patient had a previous history of chronic alcoholism and was later diagnosed with diabetes as a post-alcoholic complication of pancreatitis. He had a history of alcohol-related seizures since his late 20s and was diagnosed in his early 30s with temporal lobe epilepsy accompanied by left hippocampal sclerosis. Despite treatment with valproate $600 \mathrm{mg}$ bid, levetiracetam 1,000 $\mathrm{mg}$ bid, and vigabatrin $500 \mathrm{mg}$ bid, he was not seizure-free. He was administered $1 \mathrm{mg} / \mathrm{kg}$ intravenous lorazepam, followed by a loading dose of $15 \mathrm{mg} / \mathrm{kg}$ intravenous fosphenytoin (15 mg/kg). He continued to receive his usual doses of valproate, levetiracetam, and vigabatrin, administered through a nasogastric feeding tube. After lorazepam loading, however, his valproate dose was changed to 450 $\mathrm{mg}$ intravenous every 8 hours, and levetiracetam dose to $1,500 \mathrm{mg}$ bid, with no change in vigabatrin dose.

Although his convulsive seizures ceased, the patient did not recov- 


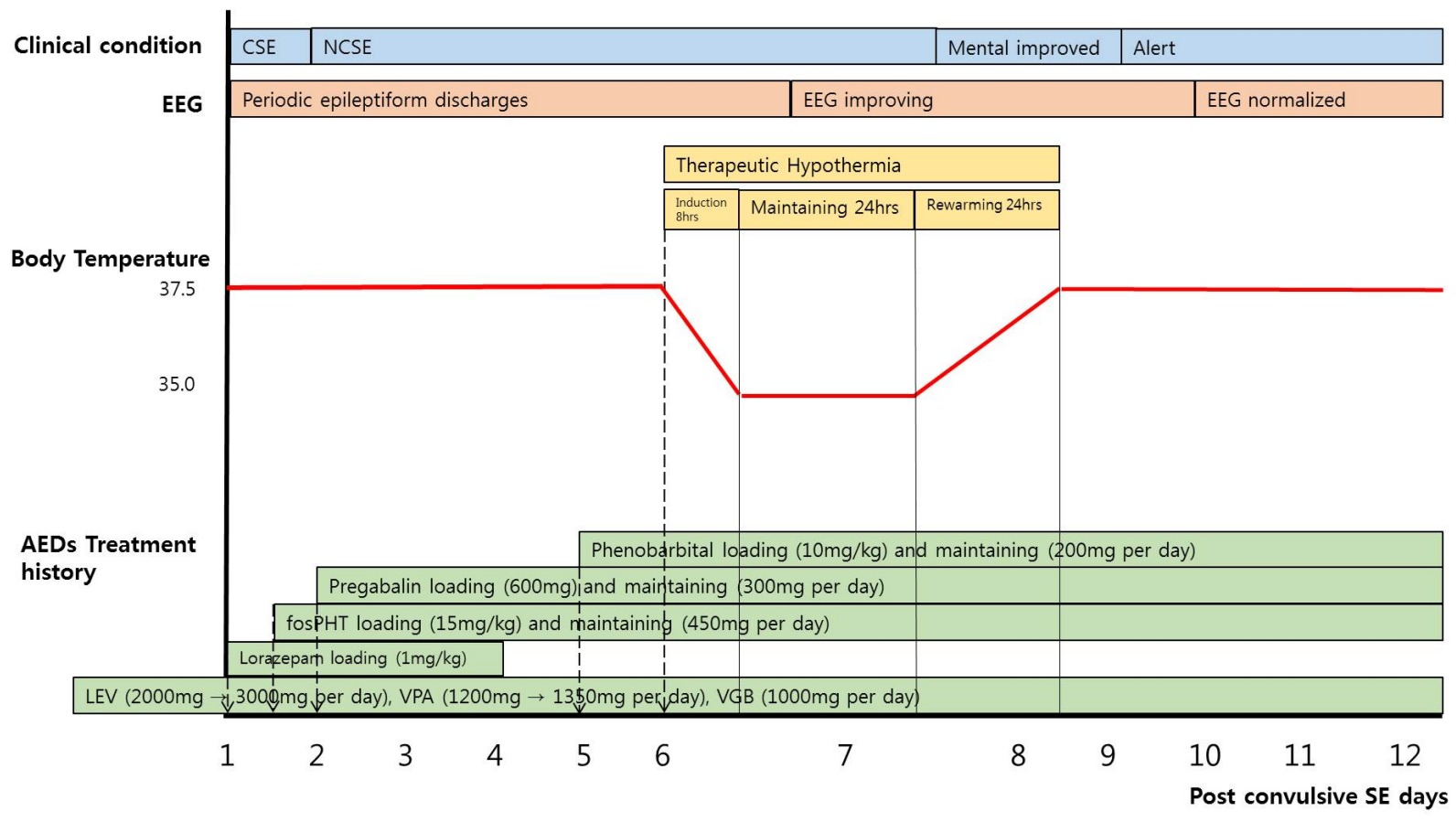

Figure 3. Summary of the clinical condition of our patient, including EEG, body temperature, and history of treatment with antiepileptic drugs. CSE, convulsive status epilepticus; NCSE, non-convulsive status epilepticus; EEG, electroencephalography; PHT, phenytoin; LEV, levetiracetam; VPA, valproic acid; VGB, vigabatrin; SE, status epilepticus.
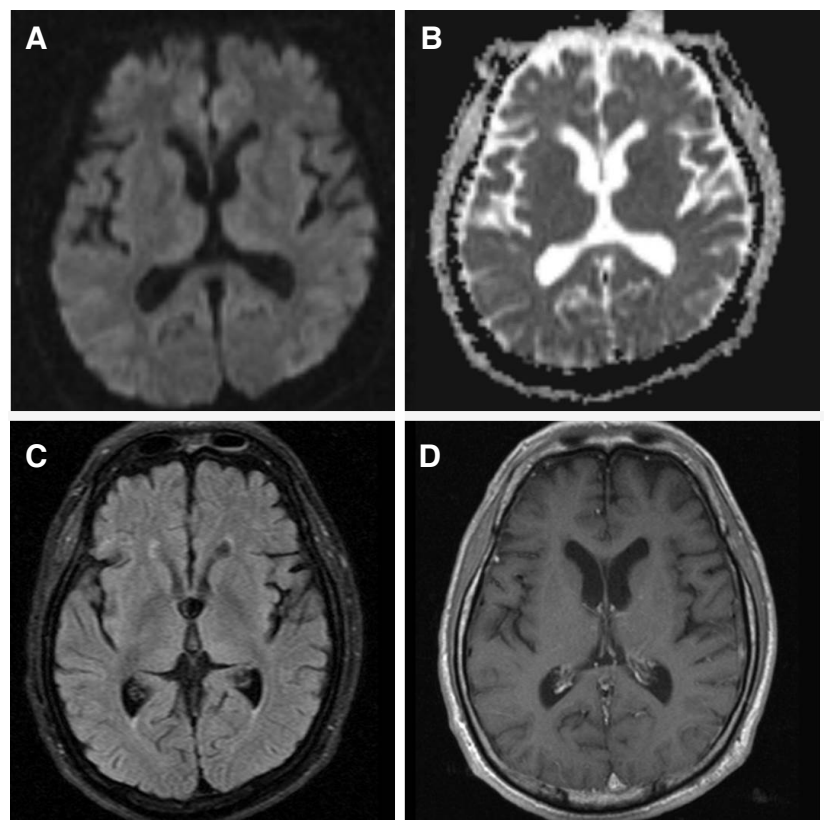

Figure 4. (A) Brain diffusion, (B) ADC, (C) fluid-attenuated inversion recovery (FLAIR), and (D) T1 enhanced MRI after 2 weeks of TH. Follow-up diffusion $M R I$ and the ADC map showed normalization of the areas with initially reduced diffusion. FLAIR and T1 enhanced MRI showed no abnormal findings. ADC, apparent diffusion coefficient; FLAIR, fluid-attenuated inversion recovery; MRI, magnetic resonance imaging; $T H$, therapeutic hypothermia. er consciousness. An initial EEG showed continuous periodic discharges in both posterior cerebral hemispheres (Fig. 1A). Diffusion-weighted magnetic resonance imaging (MRI) (Fig. 2A, B) showed symmetric diffusion-restricted lesions in the bilateral thalamus and parieto-occipital cortex with low-signal changes in apparent diffusion coefficient images (Fig. 2C, D). Several follow-up EEGs showed continuous, periodic single or poly-spikes and waves in the occipital areas (Fig. 1B). He was therefore administered loading doses of $600 \mathrm{mg}$ pregabalin and $15 \mathrm{mg} / \mathrm{kg}$ phenobarbital on days of hospital stay 2 and 5 , respectively (Fig. 3).

On day of hospital stay 6 , the patient was started on continuous EEG monitoring and $\mathrm{TH}$, without any additional medication to control seizures. At the time of starting $\mathrm{TH}$, the patient was in a semi-comatose and intubated state. $\mathrm{TH}$ at a target temperature of $35^{\circ} \mathrm{C}$ was administered using an Arctic Sun $5000^{\circledR}$ temperature management system (Medivance Arctic Sun System, Louisville, CO, USA), which uses external gel pads and circulating sterile water. The induction phase lasted 8 hours and the maintenance phase lasted 24 hours at $35^{\circ} \mathrm{C}$. The patient was monitored with esophageal core probes, and normothermia was achieved by controlled rewarming over 8 hours. The patient was not administered any benzodiazepine drugs to control shiv- 
ering or to maintain hypothermia. Rather, the patient was maintained by intravenous infusion of remifentanil hydrochloride, 2,600 $\mathrm{mg} /$ day acetaminophen and $11 \mathrm{mg} /$ day buspirone.

EEG after induction of hypothermia to $35^{\circ} \mathrm{C}$ showed that the periodic epileptiform discharges observed in this patient had decrease in amplitude without a definite change in pattern (Fig. 1C). During maintenance of hypothermia, EEG abnormalities gradually improved and the patient regained consciousness in proportion to the improvement in EEG (Fig. 1D, E). The patient was alert and his EEG normalized on the second day following initiation of the rewarming procedure. Follow-up MRI two weeks after TH showed normalization of the areas with initially-reduced diffusion (Fig. 4).

This case report was approved by the institutional review boards (IRB No. 2016-07-032) of the Sungkyunkwan University Samsung Changwon Hospital. The patient and his caregivers provided consent for publication of this report.

\section{Discussion}

After controlling generalized convulsive SE in our patient, he was successfully treated with TH for refractory NCSE. The absence of additional sedative drugs, except for remifentanil hydrochloride, during hypothermia maintenance indicates that $\mathrm{TH}$ itself may have controlled his refractory NCSE. Although TH has been used as adjunct therapy for patients with refractory epilepsy and $\mathrm{SE}_{1}^{8,11,12}$ patients with refractory convulsive SE may require sedative drugs to control shivering and maintain hypothermia. Simultaneous administration of sedative drugs limits the ability to evaluate the effects of hypothermia alone. Because our patient did not experience convulsions, he was not treated with any additional drugs that could have contributed to improvements in EEG or clinical symptoms. Findings from our patient suggest that mild TH is an appropriate treatment for refractory NCSE and should be considered before administering general anesthetics to control seizures.

The use of TH has increased worldwide, as this technique was found to be advantageous in a variety of clinical settings. Examples include unconscious adult patients with spontaneous circulation after an out-of-hospital cardiac arrest, near-drowning, traumatic head injury, hepatic encephalopathy, and stroke. ${ }^{3-5}$ Experimental studies showed that TH has anti-epileptic and neuroprotective effects, leading to its clinical use to treat $\mathrm{SE} .^{6.7}$ The neurophysiological processes involved in the anticonvulsant effects of hypothermia are still largely unclear. However, there are theoretical reasons to recommend hypo- thermia to treat SE. Hypothermia was first reported successful in treating three children with SE under thiopental anesthesia. ${ }^{8}$ Case series and case reports have shown that $\mathrm{TH}$ is safe and effective in patients with RSE. ${ }^{8,13-15}$ Complications of TH include dysrhythmias, infections, and primary coagulopathy, but these complications were reported in patients subjected to severe hypothermia $\left(<32^{\circ} \mathrm{C}\right)$, or when there were problems controlling temperature. ${ }^{3,4}$ Because mild TH has no significant adverse effects, it is regarded as relatively safe and easy to use. ${ }^{16}$ As our patient was thought to be less responsive to conventional AEDs, mild TH was deemed a possible option for managing NCSE prior to applying general anesthesia.

NCSE is defined as a state of ongoing seizures without convulsions, usually for longer than 30 minutes, associated with continuous epileptiform discharges on EEG. ${ }^{1}$ NCSE constitutes 20\% to $25 \%$ of episodes of $\mathrm{SE}^{17,18}$ and occurs in approximately $8 \%$ of all comatose patients without clinical signs of seizure activity. ${ }^{19}$ NCSE has been found to persist in $14 \%$ of patients after generalized convulsive SE is controlled. ${ }^{20}$ Although there is no generally accepted clinical definition of NCSE, early diagnosis is required to prevent potential neurological damage. Diagnosis of NCSE requires a combination of clinical and EEG features and particularly relies on EEG findings. ${ }^{21}$ Various EEG patterns have been described in NCSE, some of which clearly denote NCSE. In contrast, other patterns, such as triphasic waves, generalized periodic epileptiform discharges, and periodic lateralized epileptiform discharges (PLEDS), may not be indicative of NCSE. ${ }^{22}$ NCSE has been diagnosed in patients with PLEDs who meet certain criteria that occur in comatose patients in the aftermath of generalized tonic-clonic SE. ${ }^{21}$ Our patient was diagnosed with NCSE based on these criteria. Additionally, because the EEG and mental status of our patient gradually improved after hypothermia treatment, this improvement may also be diagnostic of NCSE.

Some NCSE case series have reported high mortality and morbidity rates, ${ }^{23-25}$ suggesting that aggressive therapy is warranted. A study of 100 patients with NCSE found that 18 had died, ${ }^{26}$ with mortality associated with acute medical causes, impairment of mental status, and development of acute complications. ${ }^{24}$ However, there is no consensus on robust treatments for NCSE, unlike SE. Therefore, treatment of NCSE depends on the type and cause. ${ }^{27}$ Because some patients with NCSE have shown more favorable outcomes than patients with convulsive $\mathrm{SE}$, caution should be used prior to further treatment with intravenous anesthetics. ${ }^{28,29}$ Aggressive pharmacological treatment appears to have a greater risk of morbidity and mortality than continuing non-convulsive seizure activity. ${ }^{30}$ Additionally, non-phar- 
macological treatments, such as hypothermia, vagal nerve stimulation, and electroconvulsive treatment, are recommended for patients with NCSE, although evidence is insufficient. Therefore, patients, such as ours, treated with various antiepileptic drugs with loading doses for a sufficient time to achieve the maximum effect, and who show no improvement in mental status or EEG may require intravenous anesthetics or other non-pharmacological treatments.

Because SE has heterogeneous causes, which greatly determine prognosis, the response of SE to treatment is difficult to evaluate using consistent and uniform criteria. However, studies have reported that $\mathrm{TH}$ has seizure cessation and reduction rates of $62.5 \%$ and $15 \%$, respectively, indicating that rate of seizure response in patients with RSE is relatively high (77.5\%). ${ }^{10}$ No correlation has been observed between target temperature and seizure response, indicating the need for additional studies on the effects, suitable target temperatures, and complications of TH. Furthermore, due to the development of TH tools with few serious adverse effects, the use of mild TH in patients with refractory NCSE should be considered in lieu of intravenous anesthetics.

\section{Conflicts of interest}

The authors have no relevant conflict of interest to report.

\section{References}

1. Kaplan PW. Nonconvulsive status epilepticus. Seminar Neuro/ 1996;16: 33-40.

2. Alroughani $R$, Javidan $M$, Qasem A, Alotaibi N. Non-convulsive status epilepticus; the rate of occurrence in general hospital. Seizure 2009;18: 38-42.

3. Nolan JP, Hazinski MF, Steen PA, Becker LB. Controversial topics from the 2005 International Consensus Conference on cardiopulmonary resuscitation and emergency cardiovascular care science with treatment recommendations. Resuscitation 2005:67:175-9.

4. Safar PJ, Kochanek PM. Therapeutic hypothermic after cardiac arrest. N Eng/ J Med 2002;346:612-3.

5. Bernard S. New indications for the use of therapeutic hypothermia. Crit Care 2004;8:E1.

6. Schmitt FC, Buchheim K, Meierkord H, Holtkamp M. Anticonvulsant properties of hypothermia in experimental status epilepticus. Neurobiol Dis 2006;23:689-96.

7. Yu L, Zhou Y, Chen W, Wang Y. Mild hypothermia pretreatment protects against pilocarpine-induced status epilepticus and neuronalapoptosis in immature rats. Neuropathology 2011;31:144-51.
8. Orlowski JP, Erenberg G, Lueders H, Cruse RP. Hypothermia and barbiturate coma for refractory status epilepticus. Crit Care Med 1984;12: 367-72.

9. Corry JJ, Dhar R, Murphy T, Diringer MN. Hypothermia for refractory status epilepticus. Neurocrit Care 2008;9:189-97.

10. Zeiler FA, Zeiler KJ, Teitelbaum J, Gillman LM, West M. Therapeutic hypothermia for refractory status epilepticus. Can J Neurol Sci 2015; 42:221-9.

11. Bennett $A E$, Hoesch $R E$, DeWitt $L D$, Afra $P$, Ansari SA. Therapeutic hypothermia for status: a report, historical perspective, and review. Clin Neuro Neurosurg 2014;126:103-9.

12. Zhumadilov A, Gilman CP, Viderman D. Management of super-refractory status epilepticus with isoflurane and hypothermia. Front Neurol 2015;5:286.

13. Bagić A, Theodore WH, Boudreau EA, et al. Towards a non-invasive interictal application of hypothermia for treating seizures: a feasibility and pilot study. Acta Neurol Scand 2008;118:240-4.

14. Cereda C, Berger MM, Rossetti AO. Bowel ischemia: a rare complication of thiopental treatment for status epilepticus. Neurocrit Care 2009:10:355-8.

15. Harbert MJ, Tam EW, Glass HC, et al. Hypothermia is correlated with seizure absence in perinatal stroke. J Child Neurol 2011;26:1126-30.

16. Hypothermia after Cardiac Arrest Study Group. Mild therapeutic hypothermia to improve the neurologic outcome after cardiac arrest. $N$ Engl I Med 2002;346:549-56.

17. Celesia GG. Modern concepts of status epilepticus. JAMA 1976;235: 1571-4.

18. Novy J, Logroscino G, Rossetti AO. Refractory status epilepticus: a prospective observational study. Epilepsia 2010;51:251-6.

19. Towne AR, Waterhouse EJ, Boggs JN, et al. Prevalence of nonconvulsive status epilepticus in comatose patients. Neurology 2000;54:340-5.

20. DeLorenzo RJ, Waterhouse EJ, Towne AR, et al. Persistent nonconvulsive status epilepticus after control of convulsive status epilepticus. Epilepsia 1998:38:833-40.

21. Sutter R, Kaplan WP. Electroencephalographic criteria for nonconvulsive status epilepticus: synopsis and comprehensive survey. Epilepsia 2012;53 Suppl 3:1-51.

22. Yu X, Shao X, Sun H, Zhong C, Cai J, Gao L. Nonconvulsive status epilepticus associated with periodic lateralized epileptiform discharges in a patient with syphilis. Interdiscip Neurosurg 2016;5:35-7.

23. Treiman DM, Delgado-Escueta AV, Clark MA. Impairment of memory following prolonged complex partial status epilepticus. Neurology 1981;31:109

24. Krumholz A, Sung GY, Fisher RS, Barry E, Bergey GK, Grattan LM. Complex partial status epilepticus accompanied by serious morbidity and mortality. Neurology 1995;45:1499-504.

25. Young GB, Jordan KG, Doig GS. An assessment of nonconvulsive seizures in the intensive care unit using continuous EEG monitoring: an investigation of variables associated with mortality. Neurology 1996;47:83-9. 
114 Journal of Epilepsy Research Vol. 7, No. 2, 2017

26. Shneker BF, Fountain NB. Assessment of acute morbidity and mortality in nonconvulsive status epilepticus. Neurology 2003;61:1066-73.

27. Meierkord $H$, Holtkamp M. Non-convulsive status epilepticus in adults: clinical forms and treatment. Lancet Neurol 2007;6:329-39.

28. Drislane FW. Presentation, evaluation, and treatment of nonconvulsive status epilepticus. Epilepsy Behav 2000;1:301-14.

29. Kaplan PW. No, some types of nonconvulsive status epilepticus cause little permanent neurologic sequelae (or: "the cure may be worse than the disease"). Neurophysiol Clin 2000;30:377-82.

30. Kaplan PW. Assessing the outcomes in patients with nonconvulsive status epilepticus: nonconvulsive status epilepticus is underdiagnosed, potentially overtreated, and confounded by comorbidity. J Clin Neurophysiol 1999; 16:341-52; discussion 353. 